\title{
UN EJEMPLO FRANCÉS DE IMITACIÓN CERVANTINA: L'HISTOIRE DES IMAGINATIONS EXTRAVAGANTES DE MONSIEUR OUFLE (1710)
}

Sobre el enmarañado tapiz de las corrientes narrativas del siglo XVII francés, un hilo discontinuo pero firme se distingue: la línea de las parodias literarias y el "antiroman», desarrollado a partir de Sorel, primer recreador francés de la locura libresca cervantina. Nuestro objetivo es analizar la relación de la obra citada del abad Bordelon principalmente con el Quijote, su modelo declarado y para ello, y a pesar de que Monsieur Oufle salga de las prensas en 1710, conviene repasar brevemente la corriente a la que ella misma se adscribe. La primera mitad del siglo XVII está dominada por la ficción narrativa idealizada: pensemos en Urfé, Gomberville, La Calprenède o los interminables romans de Mlle. de Scudéry. En paralelo se desarrolla la corriente realista y bufona auspiciada por la vieja tradición de Rabelais y relanzada por la picaresca española. Pero la del realismo no fue la única reacción que se registró contra el idealismo "romanesque». A lo largo de la centuria, rastreamos una serie de parodias de las obras más emblemáticas de cada tendencia idealista a través de la locura de un lector desenfrenado. La corriente de la «folie romanesque» crece a la sombra del Quijote, y a esa estela pertenece nuestro extravagante Monsieur Oufle. Algunos de los miembros de esta galería de lectores desquiciados habían sido Lysis, Le Berger Extravagant (1628), Le Faux Alexandre (1663), de Scarron, Le Chevalier Hypocondriaque (1632) de Saulnier o La fausse Clélie (1671) de Subligny, contrafacta burlesca de la Clélie (1654-1661) de Mlle. de Scudéry. Encontramos una ampliación del marco paródico en Les Opéras (1678) de Saint Evremont en la que Crisotine, apasionada de la ópera, se trastorna y sólo habla cantando. Y así en 1710 Bordelon publica en París los 
dos tomos de su estrafalario Monsieur Oufle, crédulo lector de tratados de magia y astrología. A pesar de que la mayor parte de su vida (1653-1730) transcurre en el siglo XVII y que sus novelistas favoritos pertenecen al mismo - Mme. de La Fayette, Scarron, La Bruyère y Cervantes ${ }^{1}$-, Bordelon es un autor ilustrado sin fisuras. Instalado plenamente en la avanzadilla ilustrada de su siglo, contemporáneo de Fontenelle y de Bayle, y admirador de Bacon y Newton y del método experimental, el resultado de su imitación cervantina y el legado de los locos librescos habían de resentirse forzosamente si es verdad, como creo que lo es, que cada época y corriente literaria actualiza en un texto heredado lo que las refleja. Poseemos escasos datos biográficos de este polígrafo en cuya obra figuran relaciones de viajes reales y fantásticos, tratados de moral cívica, reflexiones literarias, diálogos, teatro alegórico y un corpus de novelas unificadas por un propósito común: el desengaño de errores vulgares, heredados y asumidos por la falta de luces y de examen crítico del pueblo, es decir, que estamos ante un precursor de la cruzada contra el error de Feijóo. No existen estudios sistemáticos ni globales de su obra y los testimonios críticos no invitan a ello. Todos sus lectores coinciden en afirmar su mediocridad ${ }^{2}$, la pobreza de invención ${ }^{3}$ y la falta de agilidad de su pluma. En la versión reducida de la novela que ve la luz en $1789^{4}$, el editor enlaza nuestra novela con la línea de "romans critiques et satyriques» iniciada por el inimitable Quijote. Censura duramente el exceso de digresiones y justifica sus cortes: "Nous avons retouché le style et supprimé les mauvaises plaisanteries dont l'ouvrage n'est que trop abondamment rempli» (préface, p. 5) Aduce otro testimonio para justificar su decisión, el del Dictionnaire historique: «Son style est si diffus et si assommant, que les compilateurs les plus lourds trouveroient de quoi s'ennuyer» (p. 7) Y concluye su Aviso al lector con esta anécdota: «Ayant dit un jour que ses ouvrages étoient ses péchés mortels, un plaisant lui répliqua que le public en faisait pénitence» (p. 8). Maurice Bardon, el gran estudioso del cervantismo francés de los siglos XVII y XVIII, no ha sido más benévolo con él ${ }^{5}$.

1 Vid. L. Bordelon, Heures perdues du Chevalier de Rior. París, 1715, p. 95.

2 G. VAPEREAU, Dictionnaire universel des littératures. París, Hachette, 1884, p. 298.

3 L. Rius, Bibliografía crítica de la obra de M. de Cervantes. Madrid, 1899, t. II, pp. 296-297.

${ }^{4}$ Vid. M. GARNIER, Histoire de M. Oufle, en Voyages Imaginaires, romanesques, merveilleux, allégoriques, amusans, comiques et critiques, y concretamente en la serie de los «romans cabalistiques», t. 36. Amsterdam, 1789, préface.

5 M. BARDON, Don Quichotte en France au XVII et XVIII siècle. París, SlatkineChampion, 1974: "Piètre romancier» (424), «mauvais conteur» (455), «maladresse de débutant» (454). "rien n'arrête, n'émeut, ne porte à rire ou à sourire» (452). 
Pero no nos dejemos influir por este funesto preámbulo, y pasemos al texto no sin antes mencionar que a lo largo de su trayectoria narrativa, Bordelon empleó varias veces el artificio básico del Quijote: la monomanía libresca o vital. En 1708, en su Mital ou aventures incroyables, desengaño de desmanes fantásticos de las relaciones de viajes, inserta una micronarración satírica titulada Panlogue ou le sçavant hypocondriaque: "Espèce de fou qui s'est persuadé qu'il n'y a rien qu'il ne sçache" ${ }^{6}$. Al año siguiente, en 1709, publica $L e$ Voyage forcé de Bécafort, hypocondriaque, qui s'imagine être indispensablement obligé de dire ou d'écrire, et qui dit ou écrit, en effet sans aucun égard, tout ce qu'il pense des autres et de lui-même, sur quelque matière que ce soit. Y así en 1710 llegamos al fin a Monsieur Oufle. Vaya el título completo de la obra: L'Histoire des imaginations extravagantes de Monsieur Oufle causées par la lecture des livres qui traitent de la Magie, du Grimoire, des Démoniaques, Sorciers, Loupsgaroux, Incubes, Succubes et du Sabbat, des Fées, Ogres, Esprits Folets, Genies, Phantômes et autres Revenans, des Songes, de la Pierre Philosophale, de l'Astrologie Judiciaire, des Horoscopes, Talismans, Jour heureux et malheureux, Eclypses, Cometes et Almanachs, enfin de toutes les sortes d'Apparitions, de Divinations, de Sortileges, d'Enchantemens, et d'autres superstitions pratiques. Paris, chez Gosselin et Le Clerc, 1710.

En la edición de Amsterdam de ese mismo año, el editor ha añadido estratégicamente esta observación al final del título original: "écrites dans le style de Dom-Guichot" ${ }^{7}$, claro señuelo comercial.

En el prefacio, Bordelon señala la filiación de su novela a la corriente de los seres "gâtés par la lecture»" de obras tan alejadas de la verdad como de la verosimilitud: «Entre ces Histoires, les plus considerables sont celles de Dom Quixotte, du Berger extravagant et de la fausse Clélie.» (Préface, aij). M. Oufle, "pobre hombre» por el que Bordelon siente conmiseración y lástima, pasó gran parte de su vida leyendo libros de magia, fantasmas y demonios. Convencido de que lo que contenían sus libracos era totalmente cierto, renuncia a ejercer su razón crítica y vive esclavo del error y la superstición. Muchos charlatanes avispados se aprovecharon de él y otros simplemente se divirtieron a su costa. Ni argumentos, autoridades, desengaños y ni siquiera mensajes del más allá, lograron disuadirle. Bordelon presenta un aparato de notas al pie que contienen los pasajes de los libros que trastornan a Oufle en cada momento: es un alud amorfo que podemos denominar Miscelánea

\footnotetext{
6 L. Bordelon, Mital ou aventures incroyables. Paris, Le Clerc, 1708, p. viii.

7 Ed. cit. M. BARDON, op. cit., p. 449.

8 Desde este momento actualizo la ortografía. Todas las traducciones son mías.
} 
mágica y erudita, verdadero antecedente del Dictionnaire Infernal de Collin de Plancy. El frío contraste nos priva para siempre de asistir al proceso de apropiación de lo libresco por el espíritu de Oufle, asunto que a Bordelon no le interesa. La psicología del protagonista es plana. No existe más allá de su prejuicio. Su manía no es más que una excusa para alternar la miscelánea mágica con reflexiones ilustradas contra la superstición y los errores de los «esprits faibles», los «Oufles», personas esclavizadas por las opiniones ajenas. Como personaje, Oufle es un carácter monolítico, constante en sentido aristotélico y por ello esencialmente antiquijotesco ${ }^{9}$. La novela presenta una estructura deforme: el excesivo número y la extensión de sus secuencias misceláneas y ensayísticas la hacen fluctuar hacia esos géneros. Para dar una idea de la distribución narrativa del conjunto podemos dividir las secuencias narrativas en tres grupos. El primero lo componen las aventuras de Oufle basadas en su interpretación imaginaria de la realidad a partir de sus lecturas: se cree hombre-lobo, propenso a las visiones espectrales, rodeado de demonios y brujos, etc. El segundo bloque de aventuras son las derivadas de las falsas apariencias que le presentan a Oufle su mujer, sus hijos y su futuro yerno que intenta en vano vencer su manía haciéndole llegar desde el otro mundo y vía chimenea, las «Réflexions Criticomiques» contra la superstición, firmadas por «son génie». Y finalmente encontramos el tercer y más amplio componente de la novela: el digresivo. Aquí hay que incluir el aparato de notas eruditas - citas sin fin, anécdotas, cuentecillos y hasta poemas-, y también los ocho discursos sobre apariciones, la astrología judiciaria, los cometas y eclipses, las brujas, los demonios y el Sabbat, rémora de plomo para una narración poco ligera de por sí. Durante los cientos de páginas en que se desparraman, la novela es postergada sin empacho y Oufle aparcado en cualquier margen mientras Bordelon discurre cansinamente por sus alegatos contra el error. Suele utilizar un álter ego, el hermano de Oufle: el prudente y juicioso "NONCREDO». El final de la novela no desdice de su desmedrada composición. La descripción del Sabbat, nuevo centón de tópicos ${ }^{10}$, cierra la obra de forma inopinada y sin volver a

9 «Et c'est en cela que consistait son véritable caractère. Ce qu'on dira dans la suite le fera si bien connaître que j'espère qu'on ne m'accusera pas de l'avoir outré», op. cit., I,9.

10 Un nuevo caso de clara intertextualidad: siguiendo las Disquisiciones mágicas del P. DEL Río, TORRES DE VILlarRoel en su Anatomía de todo lo visible e invisible. Salamanca, Antonio de Villarroel, 1738, pp. 206-8, describe un Sabbat que por sus elementos y su disposición recuerda fuertemente al de Bordelon que, a su vez, sigue principalmente el Tableau de l'inconstance des mauvais anges et Démons de P. DE LANCRE, lector a su vez del P. del Río. 
M. Oufle. La separación se percibe tan nítidamente que en la versión reducida de 1789 , este texto aparece como apéndice.

Señalemos ya las aproximaciones a Cervantes. Desde el primer momento Bordelon se presenta como el verdadero historiador de unos hechos reales. Para incrementar la verosimilitud asegura haber desfigurado los nombres verdaderos para evitar que sean reconocidos (I,1-2) ${ }^{11}$ y nos informa de cómo ha tenido acceso a los discursos (I,153). Pretende limitar su papel al de transcriptor de unas memorias que le hicieron llegar (II,222), y aunque no cree algunas cosas de las que narra, su tarea de historiador serio le obliga a ser fiel a los hechos (II,232). Llega incluso a enjuiciar alguna de sus propias obras (II,86-87), pero la metaficción no va mucho más allá. A Bordelon también le interesó la biblioteca de Oufle, pero no lleva a cabo un escrutinio, sino que recorre los títulos de las baldas haciendo algún que otro comentario irónico: le extraña que los Oracula Magica Zoroastris se vendan como transcripción fiel del lejano Zoroastro $(\mathrm{I}, 21)$ o que algunos simples crean que el Grimoire viene firmado por su "autor», el demonio $(\mathrm{I}, 24)$.

\section{LA CRÍTICA DE LA LECTURA}

Presentar esta historia como la de un hombre que enloquece leyendo libros de magia, es desfigurar su verdadera esencia. Oufle no está loco, está confundido, instalado en el error porque no es capaz de discernir lo verdadero de lo falso. Cualidad, dicho sea de paso, muy difícil de encontrar ${ }^{12}$. Todos sus desbarres tienen una y la misma causa: el convencimiento de que lo que cuentan sus libros es absolutamente cierto. Oufle se escuda en un argumento del Quijote: la autorización y el privilegio reales. Todo cuanto él dice es necesariamente cierto porque todo «est appuyé sur des livres approuvés, privilegiés et qui par conséquent ne doivent être soupçonnés ni d'erreur ni de mensonge»(I,116-II,62;146). La misma razón que arguyó el ventero al cura cuando éste quiso convencerle de que la máquina de las caballerías era «compostura y ficción» ${ }^{13}$ y que igualmente hará suya don Quijote en la defensa de su ideal

11 Las citas corresponden al tomo y número de página.

12 Ya lo dijo La Bruyère, escritor muy admirado por Bordelon: «Après l'esprit de discernement, ce qu'il y a au monde de plus rare, ce sont les diamants et les perles». J. DE LA BRUYÈrE, Les Caractères. Paris, Flammarion, 1986, p. 315.

${ }_{13}$ «A otro perro con ese hueso (...) estando impreso con licencia de los señores del Consejo Real, como si ellos fueran gente que habían de dejar imprimir tanta mentira junta y tantas batallas, y tantos encantamientos, que quitan el juicio!». M. DE Cervantes, Don Quijote de la Mancha. Barcelona, Crítica, 1998, I, 32, p. 373. 
(I,50, 568-569). No estará de más decir que el propio Bordelon termina cayendo en su propia trampa al pedir al lector que acepte su razonamiento ya que sigue autoridades escritas muy serias y fiables, y no es una ironía (II,174).

Una sola vez en sus 797 páginas, Oufle es tildado de loco y esto, de forma aproximativa, pues se nos dice que «il pouvait passer par un véritable fou" (II,118-9). Además en varias ocasiones tiene que imponer su voluntad a su razón para amoldar su conducta a las expectativas librescas. Con todo, Bordelon critica duramente a los autores, responsables últimos de la paranoia de Oufle pues publican sin examinar críticamente sus fuentes (I,121). Pretende acabar con la vieja auctoritas de la letra escrita. Desde Descartes ninguna verdad es absoluta. El examen crítico sólo se detiene ante la Biblia, y esto de momento, pues no están hasta las explicaciones de Voltaire.

Pero si Oufle está tocado por su monomanía, otros personajes de la obra tampoco están del todo en sus casillas. Esta tendencia remite a la segunda parte del Quijote en la que se intensifica el proceso de psicotización de la sociedad en la que los cuerdos desvarían tanto o más que el loco. Pero Bordelon reconduce el mecanismo hacia la reflexión sociológica y la pertinente censura del vulgo fantasioso. Monsieur Oufle se cree hombre lobo y sale a aullar a las calles disfrazado de oso. Las reacciones de terror se suceden en los transeúntes. Pero todos dan en la fábula al narrar lo ocurrido. Bordelon analiza el fenómeno en dos planos. El primero es el individual: un jugador impaciente decide acabar con los aullidos. Al ver al lobo se le erizan los cabellos y se hiere al cerrar la puerta de la calle tremendamente asustado. Cuando el lobo se marcha, espera a recobrar el color y relata a sus compañeros de timba cómo puso en fuga a la bestia a golpes de espada y exhibe orgulloso su herida de combate $(\mathrm{I}, 62)$. En el nivel colectivo, los cuentos del vulgo no tienen fin: los que no se asomaron a la calle describían al lobo con detalles (I,75); los hubo que aseguraron que le habían cortado una pata y un mendigo manco tuvo que huir de la ciudad. Cada barrio tuvo su cuento, algunos sangrientos (I,76-77) y otros infernales (Ib.). Más tarde, tras el escándalo que Oufle montó creyendo que el perro de un carpintero era el demonio, muchos amos empezaron a desconfiar de sus fieles amigos (II,129-30). Otro desvarío flagrante es el de Sansugue, el ambicioso hijo de Oufle, que decide pedir ayuda a su padre para contactar con el Démon Barbu y lograr de éste el secreto para fabricar la piedra filosofal (II,146-147). Es decir, que Oufle no está solo. Las diatribas contra la credulidad irracional y cerril del vulgo se suceden y constituyen el principal interés del autor. Oufle es un mero protocolo de la reflexión, la crítica 
y el ensayo; un sujeto narrativo siempre rebasado puesto que Bordelon mira constantemente más allá. Su interés sociológico es prioritario y abarca los procesos de difusión y arraigo del error común, la tendencia fabuladora del vulgo, su falta de interés por ser desengañado y el poder del prejuicio entre los hombres. Oufle es el modelo de aquellos que, por no ejercer su razón crítica, sucumben a prejuicios erróneos que les esclavizan y gobiernan su vida. La invectiva ilustrada contra la falta de examen crítico es la cara inversa de la tendencia fabuladora inherente al hombre en todos los niveles y que revela el persistente gusto por lo maravilloso, verosímil o no, la divagación errática de la fantasía, rebelde a las argumentaciones.

Y al igual que Sansón Carrasco o los duques explotan la locura de don Quijote y se divierten a su costa, tampoco faltan en Bordelon actitudes semejantes. Cuando Oufle hace la corte a DULCINE, la dama le toma como bufón $(\mathrm{I}, 131)$. El resto de las burlas son interesadas y sus artífices explotan la credulidad de Oufle para lograr sus intereses. Mme Oufle decide vengarse de la curiosidad impertinente de su marido, que decidió recurrir a algunos hechizos para comprobar si ella le era infiel, usando sus mismas armas (I, 98) y el espectáculo de su pobre marido inquieto por la amistad que Belcebú le ofrece, le hace mucha gracia. En cierta ocasión Oufle sostiene que los espectros pueden robar cosas a los vivos. Ésta es la clave del plan «fantasmal» de Ruzine, la hija menor y del criado Mornand para robarle a Oufle mil luises de oro que guarda en un escritorio (I,282-3). Y además, los charlatanes se aprovechan de sus deseos de realizar los conjuros de sus libros, vendiéndole trozos de argamasa o cantos de río como las más raras y poderosas piedras mágicas. Bordelon reitera sus críticas: "Voilà comment ceux qui donnent dans les superstitions, sont presque toujours les dupes des gens habiles et adroits, qui connaissent leur faiblesse pour ne pas dire, leur sottise» $(\mathrm{I}, 101)$.

Otra reminiscencia cervantina se manifiesta en los neologismos irónicos y en las derivaciones adjetivales, marcados en cursiva: «son loup-garouïsme» (I,52); Doudou voit passer sa soeur ainsi «phantomisée» (I,279); «la raison horoscopique» $(\mathrm{I}, 299)$; «se diabolisent» (II,16); "profession talismanique» (I,418), etc.

Pasemos ahora a estudiar las relaciones del anecdotario de Bordelon. En la Miscelánea mágica y erudita figuran algunos elementos presentes en Cervantes: así, el engaño de la cabeza parlante. Bordelon describe pormenorizadamente el artificio de una cabeza de san Juan, truco extraído del Solide Trésor du petit Albert (I,248-49, nota e). Idéntico es el mecanismo de la cabeza romana con la que don Antonio Moreno burla a don Quijote y Sancho en 
II:62, pp. 1138-1142. La misma anécdota recibe un tratamiento diverso: para Bordelon está tan claro el fraude que no tiene empacho en servirse de la cabeza del evangelista o del bautista; Cervantes opta por una testa pagana y, vigilante, la hace desaparecer rápidamente ${ }^{14}$. La distancia estética e ideológica que media desde Trento a las Luces dieciochescas vuelve a aflorar al comparar los juicios sobre la astrología judiciaria y la licantropía, presentes en el Persiles, con la reflexión ilustrada de Bordelon. Soldino y Mauricio son católicos ${ }^{15} \mathrm{y}$ aficionados a la Judiciaria. El primero considera que es una ciencia que «casi enseña a adivinar» mientras que para Mauricio, que reencuentra a su hija gracias a un cálculo astrológico, se trata de una ciencia insegura, basada en la experiencia y la probabilidad (I,13; 116), argumento repetido en el Quijote, II, 25: 843, al comentar el hidalgo el poder del mono de maese Pedro. Semejantes argumentos no faltan en Bordelon $(I, 446)$, que se muestra mucho más escéptico que Cervantes cuando en el Quijote (Ib., pp.843-44), el hidalgo sostiene que es la ignorancia y falta de preparación de los que se lanzan a ella los que echan a perder «la verdad maravillosa de la ciencia» ${ }^{16}$. En cualquier caso la prudencia se impone en el Persiles $(\mathrm{I}, 18,137)$. No está de más destacar la fuerza que este cuestionamiento de la astrología cobra viniendo de Mauricio, astrólogo y razonable censor del, por momentos, inverosímil relato de Periandro (II,20, 266). Idéntico es el mecanismo de denegación que emplea Cervantes para censurar la hechicería al hacer que Cenotia reivindique su superioridad por encima de los fraudes para, desde la cima del poder mágico, afirmar que no hay hechizo que tuerza nuestro libre albedrío, lo mismo que Bordelon intenta hacer ver a Oufle para desmentir el poder absoluto de los astros y los conjuros mágicos (I,380). En definitiva el hombre pretende exculparse de responsabilidades, olvidando que cada uno es «artífice de su ventura» (Quijote, II:66, 1168), «official de su fortuna» (Silva de varia lección, 797), que «nosotros mismos nos fabricamos nuestra ventura» (Persiles, II:12, 224) y que como espeta Bordelon a los renuentes, ellos solos son «artisans de leur mauvaise

14 La misma anécdota figura en B. FeIJoo, Teatro Crítico Universal, t. II, Uso de la mágica. Madrid, BAE, 141, 1961, p.171. Es una de las múltiples coincidencias que se observan tanto en las anécdotas como en el esquema argumentativo .

15 Mauricio: I: 12, 111 y Soldino: III: 18, 393. Cito por la ed. de J. B. Avalle ARCE. Madrid, Castalia, 1992.

16 Coincido con Castro y Clemencín cuando señalan que la actitud cervantina respeta la ciencia y critica a los ignorantes y discrepo de Amezúa cuando considera que Cervantes rechaza en bloque el valor de la Judiciaria. Cf. A. CASTRo, El pensamiento de Cervantes. Barcelona, Crítica, 1987, p.101. Aunque esto no impide que se burle de la misma al atribuirle hipotéticamente a Sancho poderes de interpretación de los relinchos de las caballerías: II, 8: p. 686. 
fortune» $(\mathrm{I}, 401)$. Sin embargo hay una diferencia fundamental: mientras Cervantes acepta que las hechicerías existen y que Dios se sirve de sus efectos providencialmente (IV,10, 457), Bordelon niega taxativamente cualquier poder de la brujería más allá de la superchería o la sugestión. Y así, en el Persiles también hay una endemoniada, pero de industria, que explota la superstición popular para casarse con su amado (III,20); hay una profecía de un pueblo bárbaro, que sólo funciona como mecanismo narrativo, mientras que en Bordelon la larga serie de profecías salvajes o los falsos endemoniados, como Marta Brossier, la «romarantina», son prueba contundente de que toda la máquina de la superstición es absurda. El tratamiento de la licantropía guarda bastantes semejanzas. En el Persiles se cuenta primero la historia de Rutilio, salvado de la justicia por una hechicera mujer-lobo $(\mathrm{I}, 8,91)$, y el segundo movimiento es la reflexión al respecto del prudente Mauricio, que sostiene ser un error "grandísimo» y muy común, el creer que esto es posible. En todo caso se trata de una enfermedad, la "manía lupina» $(I, 18,134)$. Tal vez las fuentes cervantinas sean Olao Magno o Torquemada; Bordelon por su parte cita al doctor Jean Uuier para hablar de la «melancholiam item insaniam lupinam», el argumento "médico" de la licantropía extraído de De AIKAN@PSIIA morbo, quo se in lupos converti credunt homines ${ }^{17}$. Allí se cuenta el caso de un noble español que se creía convertido en oso y que se perfila como fuente de la aventura de Oufle loup-garou ${ }^{18}$. Tanto Cervantes como Bordelon citan la misma anécdota de Plinio, la historia de los árcades (Persiles, I:18, 134; y Bordelon I,33, nota c). Lo que Rutilio vio fue imaginario, ya que según el sabio Mauricio ningún ser muda su naturaleza (Ib., 134), teoría de origen agustiniano que desarrolla por extenso el francés $(I, 32)$ remontándose a los pitagóricos y a Aristóteles. Bordelon por su parte empieza por la reflexión teórica sobre la imposibilidad de la mutación y tras considerar suficientemente probado que es imposible, presenta la aventura de Oufle-loup-garou. En la más desarrollada de las fantasías librescas de Oufle, actúan numerosos mecanismos de racionalización: Oufle se cree hombre-lobo, sí, pero está bebido, está disfrazado de oso, se confunde al darse de bruces con un espejo y además su aullido no es natural, sino producto de su firme voluntad de seguir sus lecturas lupinas.

17 I. WIERI, Opera Omnia. Amsterdam, Van den Berge, 1660, pp. 335-337.

18 «6. Plinius et Edovardus scribunt, cerebrum ursi devoratum ingignere imaginationes, quasi in ursum quis transmutatus sit. Id nostro hoc seculo cuidam Hispano nobili oblatum fuisse edentum traditur, qui per deserta et montes vagabatur, se in ursum esse conversum phantasia vitiata ratus». Op. cit., p. 336-7. 


\section{CONCLUSIONES}

Genéticamente Monsieur Oufle se vincula a dos corrientes literarias dispares: si su artificio básico estructural y narrativo procede del Quijote y de la línea del antiroman francés, los contenidos concretos constituyen una buena síntesis de la abundante literatura demonológica francesa de los siglos XVI y XVII, cuyas estructuras narrativas adaptan modelos de miscelánea o de tratado doctrinal. A partir del edicto que en 1682 puso fin a la quema de brujas, la literatura demonológica cambia de orientación y empieza a repasar críticamente su propia historia. A esta línea que se prolonga en el XVIII, pertenece Monsieur Oufle, centón crítico de los tratados ya anacrónicos. En este sentido la obra de Bordelon reviste un doble interés, pues vincula la herencia cervantina no sólo al mundo ilustrado, sino también a la esfera de la demonología, al tiempo que revoluciona dicho género al presentarnos la primera versión novelizada del asunto. Y en esta hibridez, que genera los pertinentes efectos de descompensación ya comentados, reside su originalidad: Bordelon es el primer escritor que ensaya una reescritura ilustrada del Quijote que emplea como crisol en el que funde además los viejos tratados de magia con el ensayismo ilustrado. Insertando a Bordelon en su momento histórico, su Monsieur Oufle pasa de ser una novela sin gracia a convertirse en el más original de los tratados críticos sobre la magia, escrito ya en un tiempo en que es posible repasar los excesos anteriores con una media sonrisa en los labios.

Y además añade otro eslabón a la folie romanesque, y uno de los más difíciles: la «ilustración» del Quijote en el siglo que más huyó de la idealización romanesque. Las pautas de racionalización y reflexión sociológica presentes en Monsieur Oufle son las marcas de su actualidad. El resultado difícilmente puede y debe ser juzgado anacrónicamente, es decir: en relación con los antiromans del XVII. Habrá que recordar que la narrativa de dicho siglo posee un fuerte carácter de idealización y comparte el mismo universo imaginario que don Quijote y las caballerías. El mecanismo paródico-libresco de Cervantes es el cordón umbilical que mantiene el vínculo entre la nueva novela y el universo literario burlado. Esta línea de idealización invertida perdura en el Pharsamon de Marivaux (1712, aunque editada en 1737) o en Le Petit Dom Quichotte de Carmontelle, que presenta el delicioso encuentro de un don Quijote infantil y una pequeña Astrée cuyos padres los han educado a lo libresco. Valgan estos testimonios para manifestar la pervivencia de la línea paródico-idealista del XVII y saludemos al tan zaherido 
Bordelon como un pionero en la relectura cervantina y en la renovación de los cauces de la literatura demonológica. Y este doble esfuerzo no debe caer en el olvido. VALE.

CRISTINA SÁNCHEZ TALlaFigo

Universidad Complutense 